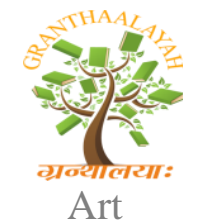

\title{
COREL PAINTER: \\ FOR THE WHOLE SKETCHING AND COLORING
}

Dr. Manorama Chouhan

Sonali Gupta

Maharani Laxmi Bai Girls P.G. College

\section{INTRODUCTION}

Corel Painter is a raster-based digital art application created to simulate as accurately as possible the appearance and behavior of traditional media associated with drawing, painting, and printmaking. It is intended to be used in real-time by professional digital artists as a functional creative tool. The current version is Painter X3, released on July 2013.

Corel Painter emulates the visual characteristics of traditional media, such as oil paint, pastel sticks, air brush, charcoal, felt pens, and other traditional artists' materials on various textured surfaces. Many of these emulated media types work with the advanced features of Wacom tablets. For instance, the airbrush tool in Painter responds to pressure as well as tilt, velocity and rotation. Corel History:

Unleashed in the early 1990s, Painter brought forth a new era for pixel based digital graphics. Painter was the first "Natural Media emulation" program, created for artists by artists! With this software, along with the newly developed

Painter was initially developed for the Macintosh system by Mark Zimmer and Tom Hedges, founders of the Fractal Design Corporation. Painter has matured over the years, surviving the transfer from its parent company, Fractal Design, to Corel Corporation, and remains unrivalled for its capacity to imitate virtually any natural medium.

At one point Painter had three companion applications: a vector-based natural media twin called Expression; a scaled-down version of Painter developed for beginners called Dabbler (later renamed Art Dabbler after the Meta creations merger); and a grayscale-only clone called Sketcher. After the Corel acquisition, Art Dabbler 2 was reintroduced as Corel Painter Essentials, now in its fourth incarnation.

In 2007 Corel released version Painter X, which was at first available in a limited edition paint can, a nod to the packaging for early releases of the software.

\section{COREL PAINTER TOOLS}

The Corel Painter application lets you draw and paint as you might with real artists' tools and media. In your studio, you use brushes, pens, pencils, chalk, airbrushes, and palette knives to make marks on a canvas or piece of paper. With Corel Painter, an infinite variety of marks are possible. Corel Painter includes a wide array of tools and features that allow you to create original artwork. Corel Painter lets you apply a wide variety of media to the canvas. For example, you can use a brush to apply colors directly from a color panel or apply a color that you mixed on the Mixer Pad. You can also paint by using a gradient, pattern, or clone.

In Corel Painter, you have the option of painting directly on the canvas by applying brushstrokes or by creating a layer and applying brushstrokes on it. Working with layers allows you to protect the canvas from any unwanted changes 


\section{INTERNATIONAL JOURNAL of RESEARCH -GRANTHAALAYAH \\ A knowledge Repository}

Art

When you draw with traditional media, the amount of pressure that you use with a tool determines the density and width of your strokes. Using a pressure-sensitive stylus with Corel Painter gives you the same kind of control. Because each artist uses a different strength or pressure level in a stroke,

The placement of objects in a painting can dramatically affect the overall appearance of the finished work. Corel Painter includes many tools and features to help you compose, size, and position images and image elements. For example, you can display the grid to help you position image elements with precision. You can also use composition tools, such as the Mirror Painting tool, to achieve visual balance.
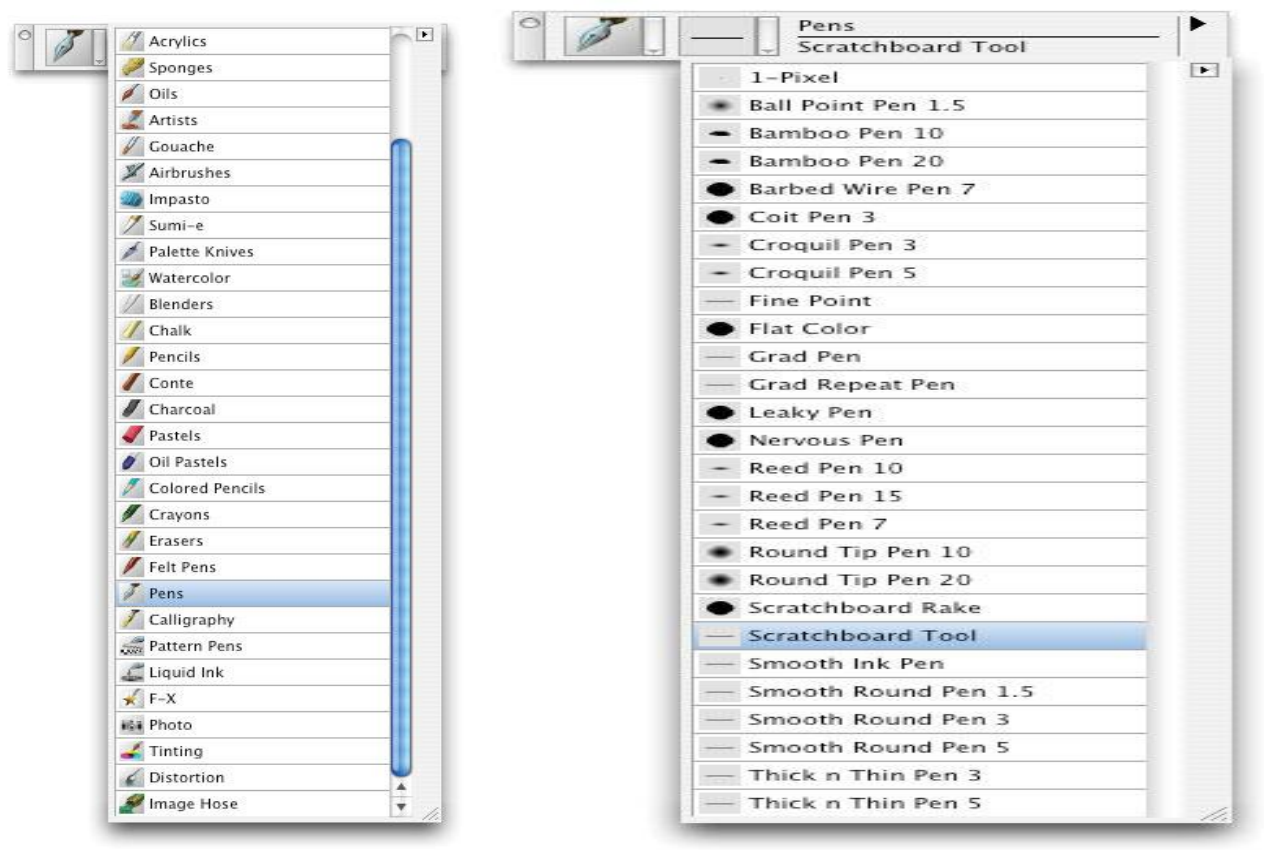

\section{PENCILS AND MARKERS AND CHALK}

Dry media, such as pencils and chalk, respond to pressure changes with variation in opacity. In real Life, overlapping pencil or crayon strokes will build up, getting Darker and denser. By contrast, chalk and pastels are opaque, so light colors can cover darker strokes. Painter uses the terms Cover and Buildup to describe these two basic Methods for determining the behavior of a brush variant.

We expect pencils and chalk to reveal the surface texture of the Paper we are using and digital dry media behaves as expected. Corel painter has different type of surface in paper selector. 


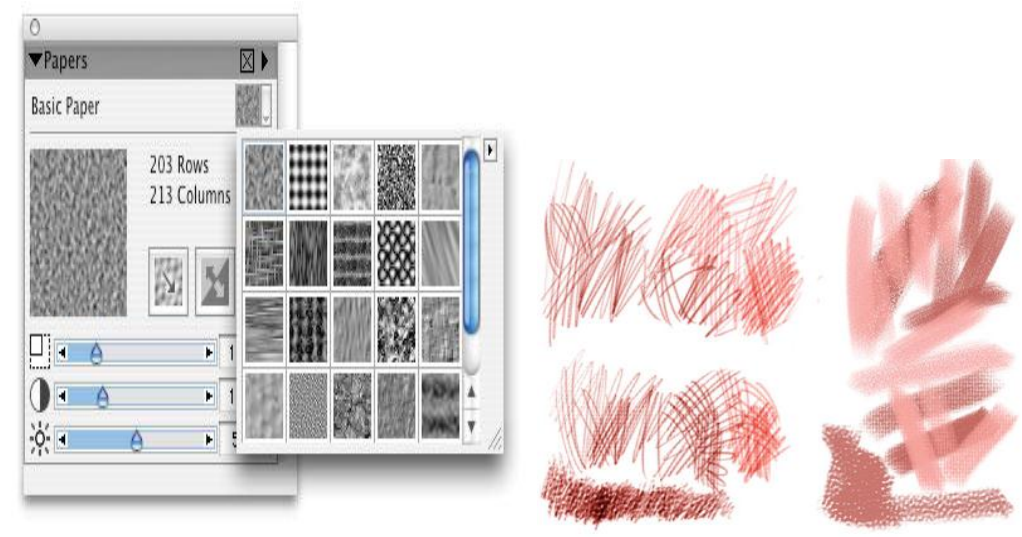

\section{REAL BRUSHES}

Traditional brushes can have a variety of shapes and are composed of numerous bristles that can range in length, thickness, and stiffness.

The kind of mark made by a brush depends on a large number Of factors: quality and number of bristles, viscosity and amount Of paint loaded, as well as pressure and direction of the artist's Stroke. There are several categories devoted to bristle-type brushes. They include Oils, Acrylic, Impasto (Italian for "thick paint"), and New in Painter X, the intriguingly named real bristle brushes.

The Bristle Oils brush seems light and airy. It shows quite a lot of Space between bristles along one edge, making for some lively Effects when strokes overlap in different directions. The bristles of Dry Clumpy Impasto are more densely packed, and we see realistic Striations in the thick paint. There is a smeary quality as well, so underlying color mixes with a new stroke. The trailing off of long Strokes is not the result of reduced pen pressure - this brush actually runs out of paint!

\section{COLOR MIXER}

The Color Mixer is available as part of the Colors Palette in the Window menu. It functions much like a traditional mixing area on an artist's palette: blobs of paint are placed next to each other, and A brush or palette knife is used to mix them partially or blend them Completely. Then the brush is loaded with a color or a combination of colors that can be applied to the canvas.

\section{THE TEMPORAL COLORS PALETTE}

The Temporal Colors palette is a floating color palette that you can display over your artwork, allowing colors to be chosen based on the colors in the image. The design and function of the Temporal Colors palette is similar to the Color panel, as they each have a Hue ring and the Saturation/Value triangle. To choose a color, click in the Hue ring and then adjust its intensity using the Saturation/Value triangle.

\section{FINE ART PRINTMAKING}

A woodcut or wood block print is a labor-intensive technique that Involves making several "plates," each carved into the surface of the wood to generate a portion of the final image. Every 
plate must be inked by hand and carefully lined up, or registered, so that it is in the proper position for pressure to be applied manually or with a Special press. The procedure is time-consuming, painstaking, and Messy You'll stay clean and relatively safe using Painter's Woodcut effect.

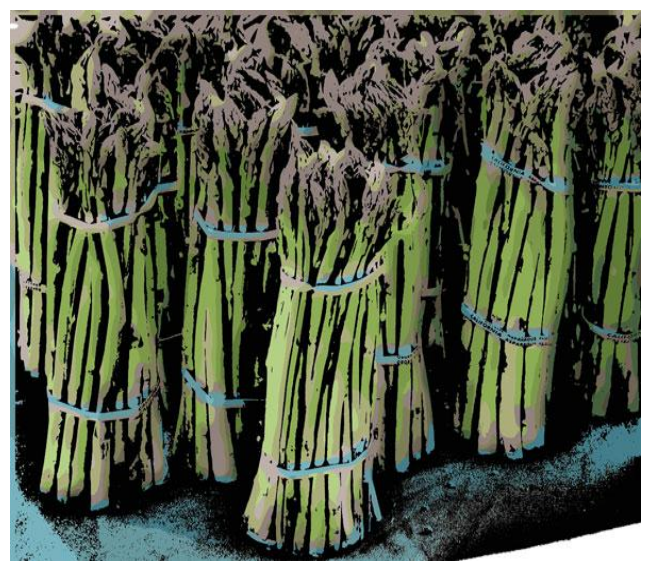

A silkscreen print, or serigraph, is created by Squeezing ink through a fine mesh onto paper or T-shirts, fabric, whatever. An image is created because parts of the mesh screen are protected from the ink with some kind of resistant fluid, or Resist. As with relief prints, several passes can be made using different screen designs and different Colors. Photographic images can be transformed into silkscreen graphics, and these are most successful when the original images are simplified. Well, Painter has a Serigraphy effect in the Surface Control group, right near the Woodcut Effect.

\section{MOBILE APP}

A new Corel Painter Mobile App has been launched on Android. The Corel Android app includes more than 70 Painter brushes, and allows artists to create and edit their artwork wherever they may be directly from their Android mobile device. Corel has also included features that enable users to import artwork directly into Painter Mobile or export using popular file formats such as JPEG, PNG or PSD.

\section{CONCLUSION}

Digital painting is huge! It's a modern creation medium that is growing rapidly and is being used in so many industries and by so many individuals' hobbyists and professionals alike. This research paper is just a slice of what's out there in the digital painting world.

Speaking of traditional media it is important to remember that digital painting is become more widely accepted and highly regarded as the techniques and resulting imagery advance.

The artist still needs to be just as talented as ever before to produce the breathtaking work. However with the benefits that digital painting offers such as increased speed freedom to experiment efficient workflow and case of sharing work with online communities. More and more artist are discovering and embracing this incredible medium.

\section{REFERENCES}

1 Rhoda Grossman. Digital Painting Fundamentals with Corel Painter X. Thomson Course Technology. Boston.

2 Susan Ruddick Bloom. (2009) Digital Painting in Photoshop. Focal Press. UK

3 Owen Demers.(2002) Digital Texturing and Painting. New Riders Publication. Unites States of America. 